\title{
WEAK UNIVERSALITY THEOREM \\ ON THE APPROXIMATION OF ANALYTIC FUNCTIONS BY SHIFTS \\ OF THE RIEMANN ZETA-FUNCTION FROM A BEATTY SEQUENCE
}

\author{
Athanasios Sourmelidis
}

\begin{abstract}
In this paper, we prove a discrete analogue of Voronin's early finite-dimensional approximation result with respect to terms from a given Beatty sequence and make use of Taylor approximation in order to derive a weak universality statement.
\end{abstract}

Communicated by Werner Georg Nowak

\section{Introduction}

Let $s=\sigma+i t \in \mathbb{C}$ (where $\sigma=\operatorname{Re}(s)$ and $t=\operatorname{Im}(s)$ ) and $\zeta(s)$ the Riemann zeta-function. This function is usually defined first on the half-plane $\{s: \operatorname{Re}(s)>1\}$ by the formula

$$
\zeta(s)=\sum_{n=1}^{\infty} \frac{1}{n^{s}}
$$

and then extended to a meromorphic function on the whole complex plane, with one simple pole at $s=1$ and no other singularity.

In 1914, H. Bohr and R. Courant [3] proved that, for any $\sigma_{0} \in\left(\frac{1}{2}, 1\right)$ the set

$$
\left\{\zeta\left(\sigma_{0}+i \tau\right): \tau \in \mathbb{R}\right\}
$$

is dense in $\mathbb{C}$. In the next year B o hr 2] proved that the same result holds for $\log \zeta\left(\sigma_{0}+i \tau\right)$. These results are called denseness theorems.

2010 Mathematics Subject Classification: 11M99, $30 \mathrm{~K} 10$.

Keywords: Universality, Riemann zeta-function, Beatty sequences. 


\section{ATHANASIOS SOURMELIDIS}

Bohr's line of investigations appears to have been almost totally abandoned for some decades. Only in 1972, S. M. V or o n in [8] obtained some significant generalizations of Bohr's denseness result.

TheOREM 1. Let $m$ be a natural number and $h$ a positive real number. For any fixed numbers $s_{1}, \ldots, s_{m}$ with $\frac{1}{2}<\operatorname{Re}\left(s_{k}\right) \leq 1$ for $1 \leq k \leq m$ and $s_{k} \neq s_{\ell}$ for $k \neq \ell$, the set

$$
\left\{\left(\zeta\left(s_{1}+i n h\right), \zeta\left(s_{2}+i n h\right), \ldots, \zeta\left(s_{m}+i n h\right)\right): n \in \mathbb{N}\right\}
$$

is dense in $\mathbb{C}^{m}$. Moreover, for any fixed number $s_{0}$ in the strip $1 / 2<\sigma \leq 1$, the set

$$
\left\{\left(\zeta\left(s_{0}+i n h\right), \zeta^{\prime}\left(s_{0}+i n h\right), \ldots, \zeta^{(m-1)}\left(s_{0}+i n h\right)\right): n \in \mathbb{N}\right\}
$$

is dense in $\mathbb{C}^{m}$.

However, V o r o $\mathrm{n}$ i n did not stop there and in 1975 proved a remarkable universality theorem for $\zeta(s)$ which states, roughly speaking, that any non-vanishing analytic function can be approximated by certain purely imaginary shifts of the zeta-function in the critical strip.

Theorem 2. Let $0<r<1 / 4$ and suppose that $g(s)$ is a non-vanishing continuous function on the disk $|s| \leq r$ which is analytic in the interior. Then, for any $\varepsilon>0$,

$$
\liminf _{T \rightarrow \infty} \frac{1}{T} \text { meas }\left\{\tau \in[0, T]: \max _{|s| \leq r}\left|\zeta\left(s+\frac{3}{4}+i \tau\right)-g(s)\right|<\varepsilon\right\}>0 .
$$

Voronin called his universality theorem the theorem about little disks. A. Rei ch [7] and B. B a g ch i [1] improved Voronin's result significantly in replacing the disk by an arbitrary compact set in the right half of the critical strip with connected complement and they even obtained a discrete analogue of it.

TheOREM 3. Suppose that $K$ is a compact subset of the strip $1 / 2<\operatorname{Re}(s)<1$ with connected complement, and let $g(s)$ be a non-vanishing continuous function on $K$ which is analytic in the interior of $K$. Then, for any $\varepsilon>0$ and any $h>0$,

$$
\liminf _{N \rightarrow \infty} \frac{1}{N} \operatorname{card}\left\{n \in \mathbb{N} \cap(0, N]: \max _{s \in K}|\zeta(s+i n h)-g(s)|<\varepsilon\right\}>0 .
$$

We note that Theorem 3 clearly implies both parts of Theorem 1 (except of $\left.\operatorname{Re}\left(s_{0}\right)=1\right)$, since the truncated Taylor series of the target function $g(s)$ can be approximated by the truncated Taylor series of a certain shift of the zetafunction. Although Theorem 1 does not suffice to prove Theorem 3, we can derive from it a weak form of universality of the zeta-function as it was first indicated by R. Garunkštis, A. La urinčikas, K. Matsumoto, J. Steuding and R. Steuding [5]. 


\section{WEAK UNIVERSALITY THEOREM}

The aim of this note is to replace the arithmetical progression $(n h)_{n \in \mathbb{N}}$ in Theorem 1 by the sequence $(\lfloor n \alpha\rfloor h)_{n \in \mathbb{N}}$ with a fixed irrational number $\alpha>0$. Here $\lfloor x\rfloor$ denotes the largest integer which is less or equal to $x$ and for given $\alpha>0$, the sequence $(\lfloor n \alpha\rfloor)_{n \in \mathbb{N}}$ is called Beatty sequence. We will consider only the case $\alpha>1$ since for $\alpha<1$ the discrete terms of the Beatty sequence is all the natural numbers and thus we get Theorem 1 . Also, $h$ will not be a random positive number but a number belonging to

where

$$
L(\alpha) \cap[0,+\infty),
$$

$L(\alpha)=\left\{h \in \mathbb{R}: 1, \alpha^{-1}, \frac{h}{2 \pi} \ln p_{1}, \frac{h}{2 \pi} \ln p_{2}, \ldots\right.$ are linearly independent over $\left.\mathbb{Q}\right\}$.

We will show later on that $L(\alpha) \cap[0,+\infty) \neq \emptyset$ for every irrational number $\alpha$.

Now, using the same arguments as $\mathrm{V}$ or on in did in [8], we will prove the following

Theorem 4 (Main theorem). Let $m$ be a natural number and $\alpha>1$ an irrational number. Let also $s_{0}, s_{1}, \ldots, s_{m}$ be fixed numbers with

$$
\frac{1}{2}<\operatorname{Re}\left(s_{k}\right) \leq 1 \text { for } 0 \leq k \leq m \text { and } s_{k} \neq s_{\ell} \text { for } k \neq \ell
$$

Then, for every $h \in L(\alpha) \cap[0,+\infty)$, the sets

and

$$
\left\{\left(\zeta\left(s_{1}+i\lfloor n \alpha\rfloor h\right), \zeta\left(s_{2}+i\lfloor n \alpha\rfloor h\right), \ldots, \zeta\left(s_{m}+i\lfloor n \alpha\rfloor h\right)\right): n \in \mathbb{N}\right\}
$$

$$
\left\{\left(\zeta\left(s_{0}+i\lfloor n \alpha\rfloor h\right), \zeta^{\prime}\left(s_{0}+i\lfloor n \alpha\rfloor h\right), \ldots, \zeta^{(m-1)}\left(s_{0}+i\lfloor n \alpha\rfloor h\right)\right): n \in \mathbb{N}\right\}
$$

are dense in $\mathbb{C}^{m}$.

Combining the preceding theorem and the method introduced in [5], we will also derive

Theorem 5 (Weak Universality). Let $\sigma_{0} \in(1 / 2,1], g: K=\overline{D\left(s_{0}, r\right)} \rightarrow \mathbb{C}$ continuous and analytic in the interior of $K$, and $\alpha>1$ irrational. Then, for every $h \in L(\alpha) \cap[0,+\infty)$ and for every $\varepsilon>0$, there exists

such that

$$
n=n(\varepsilon, h) \in \mathbb{N} \quad \text { and } \quad \delta=\delta(\varepsilon, h) \in(0,1)
$$

$$
\max _{\left|s-s_{0}\right| \leq \delta r}|\zeta(s+i\lfloor n \alpha\rfloor h)-g(s)|<\varepsilon .
$$




\section{Uniform distribution $\bmod 1$ and a set of full Lebesgue measure}

Part of the proof that Voronin gave for Theorem 1 and that we will similarly give for Theorem 4, relies on the theory of uniformly distributed sequences. A beautiful monograph on this theory is [6]. The definition, theorems and corollaries that are stated below can be found there. But before that we introduce some notation. If $\mathrm{x}=\left(x_{1}, \ldots, x_{\ell}\right) \in \mathbb{R}^{\ell}$, then $\{\mathrm{x}\}=\left(\left\{x_{1}\right\}, \ldots,\left\{x_{\ell}\right\}\right)$. Here $\left\{x_{i}\right\}$ denotes the fractional part of the real number $x_{i}$.

Definition 1. A sequence of points $\left(\mathrm{x}_{n}\right)_{n \in \mathbb{N}}$ belonging to $\mathbb{R}^{\ell}$ is said to be uniformly distributed mod 1 (u.d. $\bmod 1$ ) in $\mathbb{R}^{\ell}$ if for every box $B=I_{1} \times \cdots \times I_{\ell}$ in $[0,1]^{\ell}$ (i.e., a cartesian product of intervals), the relation

holds.

$$
\lim _{N \rightarrow \infty} \frac{\left\{1 \leq n \leq N:\left\{\mathrm{x}_{n}\right\} \in B\right\}}{N}=\left|I_{1}\right|\left|I_{2}\right| \ldots\left|I_{\ell}\right|=\operatorname{meas}(B)
$$

One of the many advantages when dealing with u.d. mod 1 sequences is a useful connection between sums and integrals, as the next theorem states.

TheOREM 6. A sequence $\left(\mathrm{x}_{n}\right)_{n \in \mathbb{N}}$ is u.d. $\bmod 1$ in $\mathbb{R}^{\ell}$ if and only if for every continuous complex-valued $f$ on $[0,1]^{\ell}$, the relation

holds.

$$
\lim _{N \rightarrow \infty} \frac{1}{N} \sum_{n=1}^{N} f\left(\left\{\mathrm{x}_{n}\right\}\right)=\int_{[0,1]^{\ell}} f(\mathrm{x}) \mathrm{dx}
$$

Pr o of. For the proof, see [6], Chapter 1, Theorem 6.1. In fact, the condition of $f$ being continuous can be relaxed to that of both $\operatorname{Re} f$ and $\operatorname{Im} f$ being Riemann integrable.

Although the multi-dimensional definition complicates somewhat the study of whether a sequence is u.d. mod 1 or not, there exists a theorem that allows us to induce the process in the one-dimensional case.

TheOREM 7. A sequence $\left(\mathrm{x}_{n}\right)_{n \in \mathbb{N}}$ is u.d. $\bmod 1$ in $\mathbb{R}^{\ell}$ if and only if for every lattice point $\mathrm{k} \in \mathbb{Z}^{\ell}, \mathrm{k} \neq \mathbf{0}$, the sequence of real numbers $\left(\left\langle\mathrm{k}, \mathrm{x}_{n}\right\rangle\right)_{n \in \mathbb{N}}$ is u.d. $\bmod 1$ in $\mathbb{R}$. Here $\langle\cdot, \cdot\rangle$ denotes the inner product as it is usually defined on the vector space $\mathbb{R}^{\ell}$.

Proof. For the proof, see [6], Chapter 1, Theorem 6.3. 
CoRollaRY 1. Let $\left(\theta_{k}\right)_{k \in \mathbb{N}}$ be a sequence of real numbers such that $1, \theta_{1}, \theta_{2}, \ldots$ are linearly independent over $\mathbb{Q}$. Then, for any $\ell \in \mathbb{N}$ and any $k_{1}, \ldots, k_{\ell} \in \mathbb{N}$ pairwise distinct, the sequence $\left(n \theta_{k_{1}}, \ldots, n \theta_{k_{\ell}}\right), n=1,2, \ldots$, is u.d. $\bmod 1$ in $\mathbb{R}^{\ell}$.

Proof. For the proof, see [6], Chapter 1, Example 6.1.

It is desirable to substitute $n$ from the above corollary with $\lfloor n \alpha\rfloor$ for given irrational $\alpha>1$. D. Carlson [4] obtained a necessary and sufficient condition for that to happen in the one-dimensional case, and with the assistance of Theorem 7 we will be able to reformulate Corollary 1 .

TheORem 8. For rational $\alpha$, the sequence $(\lfloor n \alpha\rfloor \theta)_{n \in \mathbb{N}}$ is u.d. mod 1 either for all irrationals $\theta$ or for no real number $\theta$, depending on whether $\alpha \neq 0$ or $\alpha=0$. If $\alpha$ is irrational, then $(\lfloor n \alpha\rfloor \theta)_{n \in \mathbb{N}}$ is u.d. $\bmod 1$ in $\mathbb{R}$ if and only if $1, \alpha, \alpha \theta$ are linearly independent over $\mathbb{Q}$ (or equivalently $1, \alpha^{-1}, \theta$ are linearly independent over $\mathbb{Q})$.

Proof. For the proof, see [6], Chapter 5, Theorem 1.8.

Corollary 2. Let $\alpha$ be an irrational number and $\left(\theta_{k}\right)_{k \in \mathbb{N}}$ a sequence of real numbers. Then, $1, \alpha^{-1}, \theta_{1}, \theta_{2}, \ldots$ are linearly independent over $\mathbb{Q}$ if and only if for any $\ell \in \mathbb{N}$ and any $k_{1}, \ldots, k_{\ell} \in \mathbb{N}$ pairwise distinct, the sequence $\mathbf{x}_{n}=$ $\left(\lfloor n \alpha\rfloor \theta_{k_{1}}, \ldots,\lfloor n \alpha\rfloor \theta_{k_{\ell}}\right), n=1,2, \ldots$, is $u . d . \bmod 1$ in $\mathbb{R}^{\ell}$.

Proof. The numbers $1, \alpha^{-1}, \theta_{1}, \theta_{2}, \ldots$ are linearly independent over $\mathbb{Q}$ if and only if for any $\ell \in \mathbb{N}$, any $k_{1}, \ldots, k_{\ell} \in \mathbb{N}$ pairwise distinct, and any $m_{1}, \ldots, m_{\ell} \in \mathbb{Z}$ not all of them zero, the numbers $1, \alpha^{-1}, m_{1} \theta_{k_{1}}+\cdots+m_{\ell} \theta_{k_{\ell}}$ are linearly independent over $\mathbb{Q}$. Combining Theorem 7 and Theorem 8 , we see that the latter statement is equivalent to the one saying that for any $\ell \in \mathbb{N}$ and any $k_{1}, \ldots, k_{\ell} \in \mathbb{N}$ pairwise distinct, the sequence $\mathbf{x}_{n}=\left(\lfloor n \alpha\rfloor \theta_{k_{1}}, \ldots,\lfloor n \alpha\rfloor \theta_{k_{\ell}}\right)$, $n=1,2, \ldots$, is u.d. $\bmod 1$ in $\mathbb{R}^{\ell}$.

The sequence of numbers that we are interested in is

$$
\theta_{k}=\frac{h}{2 \pi} \ln p_{k}, \quad k=1,2, \ldots,
$$

where $p_{k}$ will denote from here on the $k$ th prime and $h>0$. We prove that for a given irrational $\alpha$ there exists $h>0$ such that the necessary condition of Corollary 2 for the aforementioned sequence is fulfilled. In fact we prove the existence of a lot such $h$.

TheOREM 9. Let $\alpha$ be an irrational number and $L(\alpha)=\left\{h \in \mathbb{R}: 1, \alpha^{-1}, \frac{h}{2 \pi} \ln p_{1}, \frac{h}{2 \pi} \ln p_{2}, \ldots\right.$ are linearly independent over $\left.\mathbb{Q}\right\}$. 
The set $L(\alpha)$ has full Lebesgue measure in $\mathbb{R}$, i.e., meas $(\mathbb{R} \backslash L(\alpha))=0$.

Pr o of. Let $B=\mathbb{R} \backslash L(\alpha)$ and $h \in B$. Then, the numbers

$$
1, \alpha^{-1}, \quad \frac{h}{2 \pi} \ln p_{1}, \quad \frac{h}{2 \pi} \ln p_{2}, \ldots
$$

are linearly dependent over $\mathbb{Q}$ and consequently over $\mathbb{Z}$ as well. Thus, there exists integer $k \geq 1$ and integers $a_{1}, \ldots, a_{k}, b, c$, where $a_{i}$ are not all zeros, such that

$$
a_{1} \frac{h}{\pi} \ln p_{1}+\cdots+a_{k} \frac{h}{\pi} \ln p_{k}=b+c \alpha^{-1} .
$$

Putting $A=p_{1}^{a_{1}} \ldots p_{k}^{a_{k}}$, we observe that $A \in \mathbb{Q}^{+} \backslash\{1\}$ and we can rewrite (1) as

$$
h \ln A=b \pi+c \alpha^{-1} \pi .
$$

Fix a vector $(A, b, c) \in\left(\mathbb{Q}^{+} \backslash\{1\}\right) \times \mathbb{Z} \times \mathbb{Z}=\Gamma$. Consider the corresponding set

$$
B(A, b, c)=\left\{h \in \mathbb{R}: h \ln A=b \pi+c \alpha^{-1} \pi\right\} .
$$

The set $B(A, b, c)$ is clearly a singleton (since $\ln A \neq 0$ ) and thus of measure zero. Hence, the countable union of singletons

$$
B=\bigcup_{(A, b, c) \in \Gamma} B(A, b, c)
$$

is of measure zero. Therefore, its complement $\mathbb{R} \backslash B=L(\alpha)$ has full Lebesgue measure in $\mathbb{R}$.

\section{Auxiliary lemmas}

Before stating the lemmas needed for the proofs of Theorems 4 and 5 , we introduce some notation. Let $\Omega$ denote the set of all sequences of real numbers indexed by the prime numbers in ascending order. Further, define for every finite subset $M$ of the set of all primes, every $\omega=\left(\omega_{2}, \omega_{3}, \omega_{5}, \ldots\right) \in \Omega$, and all complex numbers $s$, the truncated Euler product

$$
\zeta_{M}(s, \omega)=\prod_{p \in M}\left(1-\frac{\exp \left(-2 \pi i \omega_{p}\right)}{p^{s}}\right)^{-1} .
$$

Obviously, $\zeta_{M}(s, \omega)$ is a non-vanishing analytic function of $s$ in the half-plane $\sigma>0$. Observe also that for $M=\left\{p_{1}^{\prime}, \ldots, p_{\ell}^{\prime}\right\}$ and constant $s, \zeta_{M}(s, \omega)$ can be treated as a continuous complex-valued function of $\ell$ variables $\left(\omega_{p_{1}^{\prime}}, \ldots, \omega_{p_{\ell}^{\prime}}\right)$ defined on $[0,1]^{\ell}$. In such cases, where $M$ and $s$ are given, $\zeta_{M}(s, \omega)$ will be abbreviated as $\zeta_{M}\left(s, \omega_{p_{1}^{\prime}}, \ldots, \omega_{p_{\ell}^{\prime}}\right)$. Finally, $\log z$ will denote the principal logarithm of $z$. 


\section{WEAK UNIVERSALITY THEOREM}

LEMma 1. Let $s_{0}$ be complex number such that $\frac{1}{2}<\operatorname{Re}\left(s_{0}\right) \leq 1$ and $k \in \mathbb{N}_{0}$. If we define $M_{Q}=\left\{p_{1}, p_{2}, \ldots, p_{Q}\right\}$ to be the set of the first $Q$ primes and $\mathbf{0}=(0,0, \ldots)$, then

$$
\lim _{Q \rightarrow \infty} \limsup _{T \rightarrow \infty} \frac{1}{T} \int_{0}^{T}\left|\zeta^{(k)}\left(s_{0}+i t\right)-\zeta_{M_{Q}}^{(k)}\left(s_{0}+i t, \mathbf{0}\right)\right|^{2} d t=0 .
$$

Pr o of. For the proof, see [8], pages 164-166, 168.

Lemma 2. Suppose that $\left(a_{1}, \ldots, a_{m}\right) \in \mathbb{C}^{m}, \varepsilon>0, y \in \mathbb{N}$ and $x_{p_{1}}, \ldots, x_{p_{y}}$ are real numbers, and $s_{1}, \ldots, s_{m}$ are the numbers in the condition of Theorem 4, where $\operatorname{Im}\left(s_{k}\right)>2$ for all $1 \leq k \leq m$. Then, there exists a finite set of primes $M=\left\{p_{1}^{\prime}, p_{2}^{\prime}, \ldots, p_{\ell}^{\prime}\right\}$ and a sequence $\omega \in \Omega$ such that

$$
M \supset\left\{p_{1}, \ldots, p_{y}\right\}, \quad \omega_{p_{r}}=x_{p_{r}} \quad \text { and } \quad\left|\zeta_{M}\left(s_{k}, \omega\right)-a_{k}\right|<\varepsilon,
$$

for $1 \leq r \leq y$ and $1 \leq k \leq m$.

Pr o of. For the proof, see [8], Lemma 11.

Lemma 3. Suppose that $\left(a_{0}, \ldots, a_{m-1}\right) \in \mathbb{C}^{m}, \varepsilon>0, y \in \mathbb{N}$ and $x_{p_{1}}, \ldots, x_{p_{y}}$ are real numbers, and $s_{0}$ is a number with $\frac{1}{2}<\operatorname{Re}\left(s_{0}\right) \leq 1$ and $\operatorname{Im}\left(s_{0}\right)>2$. Then, there exists a finite set of primes $M=\left\{p_{1}^{\prime}, p_{2}^{\prime}, \ldots, p_{\ell}^{\prime}\right\}$ and a sequence $\omega \in \Omega$ such that

$$
M \supset\left\{p_{1}, \ldots, p_{y}\right\}, \quad \omega_{p_{r}}=x_{p_{r}} \quad \text { and } \quad\left|\zeta_{M}^{(k)}\left(s_{0}, \omega\right)-a_{k}\right|<\varepsilon,
$$

for $1 \leq r \leq y$ and $1 \leq k \leq m$.

Pr o of. For the proof, see [8], Lemma 12.

REMARK 1. Note that the condition for the imaginary parts of the complex numbers in Lemma 2 can be removed:

P r o of. Let the assumptions of Lemma 2 hold without the restriction of the imaginary parts. There exists a number $c>0$ such that $\operatorname{Im}\left(s_{k}\right)+2 \pi c>2$ for $1 \leq k \leq m$. According to Lemma 2 , for $\tilde{x}_{p_{1}}=x_{p_{1}}-c \ln p_{1}, \ldots, \tilde{x}_{p_{y}}=x_{p_{y}}-c \ln p_{y}$, there exists a finite set of primes $M$ and $\tilde{\omega} \in \Omega$ such that

$$
M \supset\left\{p_{1}, \ldots, p_{y}\right\}, \quad \tilde{\omega}_{p_{r}}=\tilde{x}_{p_{r}} \text { and } \quad\left|\zeta_{M}\left(s_{k}+2 \pi i c, \tilde{\omega}\right)-a_{k}\right|<\varepsilon .
$$

for $1 \leq r \leq y$ and $1 \leq k \leq m$. 


\section{ATHANASIOS SOURMELIDIS}

Taking $\omega \in \Omega$ to be $\omega_{p}=\tilde{\omega}_{p}+c \operatorname{lnp}$ for all primes $p$, we observe that for $1 \leq k \leq m$,

$$
\begin{aligned}
\zeta_{M}\left(s_{k}+2 \pi i c, \tilde{\omega}\right) & =\prod_{p \in M}\left(1-\frac{\exp \left(-2 \pi i \tilde{\omega}_{p}\right)}{p^{s_{k}+2 \pi i c}}\right)^{-1} \\
& =\prod_{p \in M}\left(1-\frac{\exp \left(-2 \pi i\left(\tilde{\omega}_{p}+c \ln p\right)\right.}{p^{s_{k}}}\right)^{-1} \\
& =\prod_{p \in M}\left(1-\frac{\exp \left(-2 \pi i \omega_{p}\right)}{p^{s_{k}}}\right)^{-1} \\
& =\zeta_{M}\left(s_{k}, \omega\right)
\end{aligned}
$$

and of course, $\omega_{p_{r}}=x_{p_{r}}$ for $1 \leq r \leq y$.

REMARK 2. The same result as above can be obtained similarly for Lemma 3, since to prove it Vor o n in showed that the set of points

$$
\Delta_{(M, \omega)}=\left(\log \zeta_{M}\left(s_{0}, \omega\right),\left[\log \zeta_{M}\left(s_{0}, \omega\right)\right]^{\prime}, \ldots,\left[\log \zeta_{M}\left(s_{0}, \omega\right)\right]^{(m-1)}\right) \in \mathbb{C}^{m}
$$

is dense in $\mathbb{C}^{m}$ whenever $(M, \omega)$ runs through all possible finite sets of primes $M$ and $\omega \in \Omega$ with the requirements $M \supset\left\{p_{1}, \ldots, p_{y}\right\}$ and $\omega_{p_{r}}=x_{p_{r}}$ for $1 \leq r \leq y$.

Lemma 4. Let $t_{0}, t_{1}, \ldots, t_{R}$ be real numbers, where $t_{0}<t_{1}<\cdots<t_{R}$. If $G(t)$ is a complex-valued function which is defined and continuously differentiable on the interval $\left[t_{0}, t_{R}\right]$, then

$$
\sum_{r=1}^{R}\left|G\left(t_{r}\right)\right|^{2} \leq \frac{1}{\delta} \int_{t_{0}}^{t_{R}}|G(t)|^{2} \mathrm{~d} t+2\left(\int_{t_{0}}^{t_{R}}|G(t)|^{2} \mathrm{~d} t\right)^{\frac{1}{2}}\left(\int_{t_{0}}^{t_{R}}\left|G^{\prime}(t)\right|^{2} \mathrm{~d} t\right)^{\frac{1}{2}}
$$

where

$$
\delta=\min _{0 \leq r<R}\left|t_{r+1}-t_{r}\right|
$$

Pr o of. For the proof, see 8], Lemma 6.

LEMMA 5. Let $s_{1}, \ldots, s_{\ell}$ be numbers such that $\operatorname{Re}\left(s_{j}\right)>0$ for $j=1, \ldots, \ell$, and $m \in \mathbb{N}$. Then, for every $\varepsilon>0$, there exists an $N=N(\varepsilon) \in \mathbb{N}$ such that for every set $S$ of prime numbers greater than $p_{N}$, every $k=0,1, \ldots, m$, every $j=1, \ldots, \ell$ and every $\omega \in \Omega$, the inequality

holds.

$$
\left|\left(\zeta_{S}\left(s_{j}, \omega\right)-1\right)^{(k)}\right|<\varepsilon
$$


Proof. Let $\varepsilon>0$ and

$$
0<t_{0}<r_{0}<\min _{1 \leq j \leq \ell} \operatorname{Re}\left(s_{j}\right)
$$

If we set

$$
\varepsilon^{\prime}=\min _{0 \leq k \leq m} \frac{\varepsilon r_{0}^{k}}{k !},
$$

then there exists a $\delta=\delta(\varepsilon)<1$ such that $\left|e^{z}-1\right|<\varepsilon^{\prime}$ for every $|z|<\delta$. Since the series

$$
\sum_{n=1}^{\infty} \frac{1}{p_{n}^{t_{0}}-1}
$$

converges, there exists an $N=N(\varepsilon)$ such that

$$
\sum_{n=N}^{\infty} \frac{1}{p_{n}^{\sigma}-1}<\frac{\delta}{2}
$$

for every $\sigma>t_{0}$. Now let $S$ be a set of prime numbers greater than $p_{N}$ and $\omega \in \Omega$. Observe that whenever $|z|<\frac{1}{2}$, one can obtain

$$
|\log (1+z)|=\left|\int_{1}^{1+z} \frac{\mathrm{d} w}{w}\right| \leq \int_{1}^{1+z} \frac{|\mathrm{d} w|}{|w|} \leq 2|z| .
$$

Keeping that in mind and taking advantage of the fact that for every $\operatorname{Re}(s)>t_{0}$ and $n \geq N$ :

$$
\begin{aligned}
\left|\left(1-\frac{\exp \left(-2 \pi i \omega_{p_{n}}\right)}{p_{n}^{s}}\right)^{-1}-1\right| & =\left|\frac{\exp \left(-2 \pi i \omega_{p_{n}}\right)}{p_{n}^{s}-\exp \left(-2 \pi i \omega_{p_{n}}\right)}\right| \\
& \leq \frac{1}{p_{n}^{\sigma}-1}<\frac{\delta}{2}<\frac{1}{2}
\end{aligned}
$$

we can estimate

$$
\begin{aligned}
\left|\sum_{p \in S} \log \left(1-\frac{\exp \left(-2 \pi i \omega_{p}\right)}{p^{s}}\right)^{-1}\right| & \leq \sum_{p \in S}\left|\log \left(1-\frac{\exp \left(-2 \pi i \omega_{p}\right)}{p^{s}}\right)^{-1}\right| \\
& \leq 2 \sum_{n=N}^{\infty}\left|\left(1-\frac{\exp \left(-2 \pi i \omega_{p_{n}}\right)}{p_{n}^{s}}\right)^{-1}-1\right| \\
& \leq 2 \sum_{n=N}^{\infty} \frac{1}{p_{n}^{\sigma}-1}<\delta .
\end{aligned}
$$


Thus, for every $\operatorname{Re}(s)>t_{0}$,

$$
\left|\zeta_{S}(s, \omega)-1\right|=\left|\exp \left(\sum_{p \in S} \log \left(1-\frac{\exp \left(-2 \pi i \omega_{p}\right)}{p^{s}}\right)^{-1}\right)-1\right|<\varepsilon^{\prime} .
$$

All inequalities

$$
\left|\left(\zeta_{S}\left(s_{j}, \omega\right)-1\right)^{(k)}\right|<\varepsilon,
$$

for $k=0, \ldots, m$ and $j=1, \ldots, \ell$, can now be proved by computing the Cauchy's estimates of $\zeta_{S}(s, \omega)-1$ on the disks $D\left(s_{j}, r_{0}\right) \subset\left\{s: \operatorname{Re}(s)>t_{0}\right\}$, for $j=1, \ldots, \ell$, respectively.

\section{Proofs of Theorem 4 and Theorem 5}

Proof of The or e m 4. We prove the second part of Theorem 4 since the first part can be shown similarly. Let $s_{0}$ be a complex number with $\frac{1}{2}<\operatorname{Re}\left(s_{0}\right) \leq 1$, $\alpha>1$ irrational and $h \in L(\alpha) \cap[0,+\infty)$, where $L(\alpha)$ is the set defined in the first section and in Theorem 9 .

To prove the theorem it suffices to show that any vector $\left(a_{0}, \ldots, a_{m-1}\right) \in \mathbb{C}^{m}$ can be approximated arbitrarily close by the vector

$$
\left(\zeta\left(s_{0}+i\lfloor n \alpha\rfloor h\right), \ldots, \zeta^{(m-1)}\left(s_{0}+i\lfloor n \alpha\rfloor h\right)\right)
$$

with a suitable natural number $n$. We fix any $\left(a_{0}, \ldots, a_{m-1}\right)$. By Lemma 3 , for every $\varepsilon>0$ and every $y \in \mathbb{N}$, there exists $\zeta_{M}\left(s_{0}, \omega\right)$ such that $M \supset\left\{p_{1}, \ldots, p_{y}\right\}$, $\omega_{p_{r}}=0$ for $1 \leq r \leq y$, and for $k=0, \ldots, m-1$ we have

$$
\left|\zeta_{M}^{(k)}\left(s_{0}, \omega\right)-a_{k}\right|<\varepsilon .
$$

Let $M=\left\{p_{1}^{\prime}, \ldots, p_{\ell}^{\prime}\right\}$. By the continuity of $\zeta_{M}\left(s_{0}, \omega_{p_{1}^{\prime}}, \ldots, \omega_{p_{\ell}^{\prime}}\right)$ as a function of $\ell$ variables and (2), in $[0,1]^{\ell}$ there exists a subbox $K$ with meas $(K)>0$ such that for $k=0, \ldots, m-1$ all the points $\left(x_{p_{1}^{\prime}}, \ldots, x_{p_{\ell}^{\prime}}\right)$ belonging in $K$ satisfy

$$
\left|\zeta_{M}^{(k)}\left(s_{0}, x_{p_{1}^{\prime}}, \ldots, x_{p_{\ell}^{\prime}}\right)-a_{k}\right|<2 \varepsilon .
$$

Let $\sum_{n=1}^{\prime N}$ denote summation over those $n \in[1, N] \cap \mathbb{N}$ for which

$$
\left(\left\{\frac{h \ln p_{1}^{\prime}}{2 \pi}\lfloor n \alpha\rfloor\right\}, \ldots,\left\{\frac{h \ln p_{\ell}^{\prime}}{2 \pi}\lfloor n \alpha\rfloor\right\}\right) \in K .
$$


We consider the expression

$$
A_{N}=\frac{1}{N} \sum_{n=1}^{N} \sum_{k=0}^{m-1}\left|\zeta^{(k)}\left(s_{0}+i\lfloor n \alpha\rfloor h\right)-\zeta_{M}^{(k)}\left(s_{0}+i\lfloor n \alpha\rfloor h, \mathbf{0}\right)\right|^{2} .
$$

We choose $Q$ larger than any $p \in M$ and we define $M_{Q}=\left\{p_{1}, p_{2}, \ldots, p_{Q}\right\}$ to be the set of the first $Q$ primes. Then,

$$
\begin{aligned}
A_{N} \leq & \frac{2}{N} \sum_{n=1}^{N} \sum_{k=0}^{m-1}\left|\zeta^{(k)}\left(s_{0}+i\lfloor n \alpha\rfloor h\right)-\zeta_{M_{Q}}^{(k)}\left(s_{0}+i\lfloor n \alpha\rfloor h, \mathbf{0}\right)\right|^{2} \\
& +\frac{2}{N} \sum_{n=1}^{N} \sum_{k=0}^{\prime}\left|\zeta_{M_{Q}}^{(k)}\left(s_{0}+i\lfloor n \alpha\rfloor h, \mathbf{0}\right)-\zeta_{M}^{(k)}\left(s_{0}+i\lfloor n \alpha\rfloor h, \mathbf{0}\right)\right|^{2} .
\end{aligned}
$$

We denote the first double sum by $S_{1}$ and the second by $S_{2}$. Firstly, we estimate $S_{2}$. We make use of Leibniz's formula

$$
\zeta_{M_{Q}}^{(k)}-\zeta_{M}^{(k)}=\left[\zeta_{M}\left(\zeta_{M_{Q} \backslash M}-1\right)\right]^{(k)}=\sum_{j=0}^{k}\left(\begin{array}{c}
k \\
j
\end{array}\right) \zeta_{M}^{(j)}\left(\zeta_{M_{Q} \backslash M}-1\right)^{(k-j)} .
$$

Applying the Cauchy-Schwarz inequality, we obtain

$$
\left|\zeta_{M_{Q}}^{(k)}-\zeta_{M}^{(k)}\right|^{2} \leq(k+1) \sum_{j=0}^{k}\left|\left(\begin{array}{c}
k \\
j
\end{array}\right) \zeta_{M}^{(j)}\left(\zeta_{M_{Q} \backslash M}-1\right)^{(k-j)}\right|^{2} .
$$

Hence, putting in $S_{2}$ the summation over $n$ on the inside, we get

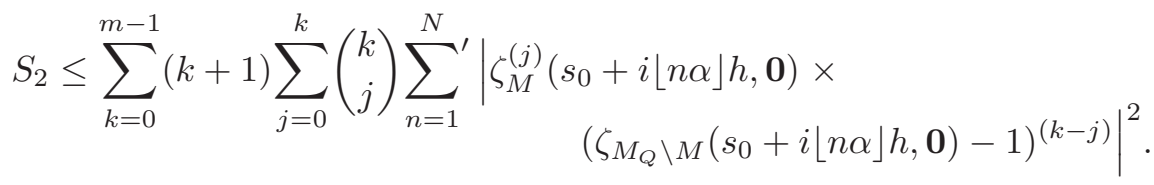

So it suffices to estimate the sums of the form

$$
S_{k, j}=\sum_{n=1}^{N}\left|\zeta_{M}^{(j)}\left(s_{0}+i\lfloor n \alpha\rfloor h, \mathbf{0}\right)\left(\zeta_{M_{Q} \backslash M}\left(s_{0}+i\lfloor n \alpha\rfloor h, \mathbf{0}\right)-1\right)^{(k-j)}\right|^{2} .
$$

Note that if $M_{Q} \backslash M=\left\{p_{1}^{\prime \prime}, \ldots, p_{Q-\ell}^{\prime \prime}\right\}$, then a simple computation leads to

$$
\zeta_{M}\left(s_{0}+i\lfloor n \alpha\rfloor h, \mathbf{0}\right)=\zeta_{M}\left(s_{0},\left\{\frac{h \ln p_{1}^{\prime}}{2 \pi}\lfloor n \alpha\rfloor\right\}, \ldots,\left\{\frac{h \ln p_{\ell}^{\prime}}{2 \pi}\lfloor n \alpha\rfloor\right\}\right)
$$

and

$$
\zeta_{M_{Q} \backslash M}\left(s_{0}+i\lfloor n \alpha\rfloor h, \mathbf{0}\right)=\zeta_{M_{Q} \backslash M}\left(s_{0},\left\{\frac{h \ln p_{1}^{\prime \prime}}{2 \pi}\lfloor n \alpha\rfloor\right\}, \ldots,\left\{\frac{h \ln p_{Q-\ell}^{\prime \prime}}{2 \pi}\lfloor n \alpha\rfloor\right\}\right) .
$$


We define $F:[0,1]^{Q} \rightarrow \mathbb{C}$ to be of the form

$$
\begin{aligned}
F\left(\omega_{p_{1}}, \ldots, \omega_{p_{Q}}\right)= & \mid \zeta_{M}^{(j)}\left(s_{0}, \omega_{p_{1}^{\prime}}, \ldots, \omega_{p_{\ell}^{\prime}}\right) \\
& \times\left.\left(\zeta_{M_{Q} \backslash M}\left(s_{0}, \omega_{p_{1}^{\prime \prime}}, \ldots, \omega_{p_{Q-\ell}^{\prime \prime}}\right)-1\right)^{(k-j)}\right|^{2},
\end{aligned}
$$

whenever $\left(\omega_{p_{1}^{\prime}}, \ldots, \omega_{p_{\ell}^{\prime}}\right) \in K$, and zero otherwise. If we set

$$
\mathbf{x}_{n}=\left(\frac{h \ln p_{1}}{2 \pi}\lfloor n \alpha\rfloor, \ldots, \frac{h \ln p_{Q}}{2 \pi}\lfloor n \alpha\rfloor\right), \quad n \in \mathbb{N},
$$

then

$$
S_{k, j}=\sum_{n=1}^{N} F\left(\left\{\mathrm{x}_{n}\right\}\right)=\sum_{n=1}^{N} F\left(\left\{\mathrm{x}_{n}\right\}\right) .
$$

The last equality is true if we consider the definitions of $\sum^{\prime}$ and $F$. Now recall that $h \in L(\alpha)$. Thus, according to Corollary 2 , the sequence $\left(\mathrm{x}_{n}\right)_{n \in \mathbb{N}}$ is u.d. $\bmod$ 1 in $\mathbb{R}^{Q}$. Using Theorem 6 , we obtain

$$
\begin{aligned}
\lim _{N \rightarrow \infty} \frac{1}{N} S_{k, j}= & \lim _{N \rightarrow \infty} \frac{1}{N} \sum_{n=1}^{N} F\left(\left\{\mathrm{x}_{n}\right\}\right)=\int_{[0,1]^{Q}} F(\mathrm{x}) \mathrm{d} \mathrm{x}=\int_{K} \int_{[0,1]^{Q-\ell}} F(\mathrm{x}) \mathrm{d} \mathrm{x} \\
= & \int_{K}\left|\zeta_{M}^{(j)}\left(s_{0}, \omega_{p_{1}^{\prime}}, \ldots, \omega_{p_{\ell}^{\prime}}\right)\right|^{2} \mathrm{~d} \omega_{p_{1}^{\prime}} \ldots \mathrm{d} \omega_{p_{\ell}^{\prime}} \\
& \times \int_{[0,1]^{Q-\ell}}\left|\left(\zeta_{M_{Q} \backslash M}\left(s_{0}, \omega_{p_{1}^{\prime \prime}}, \ldots, \omega_{p_{Q-\ell}^{\prime \prime}}\right)-1\right)^{(k-j)}\right|^{2} \mathrm{~d} \omega_{p_{1}^{\prime \prime}} \ldots \mathrm{d} \omega_{p_{Q-\ell}^{\prime \prime}} .
\end{aligned}
$$

By (3), the first integral is bounded by $\left(\left|a_{j}\right|+2 \varepsilon\right)^{2}$ meas $(K)$, and the second integral, in view of Lemma 5, approaches zero uniformly in $Q$ as $y$ increases. Hence, by (5) and (6), we may choose $y$ sufficiently large so that for every $Q$ larger than any $p \in M$, we can find an $N_{0}=N_{0}(Q)$ with the property

We estimate $S_{1}$,

$$
S_{2}<N \operatorname{meas}(K) \frac{\varepsilon^{3}}{2} \quad \text { for } N \geq N_{0}
$$

$$
S_{1}=\sum_{k=0}^{m-1} \sum_{n=1}^{N}\left|\zeta^{(k)}\left(s_{0}+i\lfloor n \alpha\rfloor h\right)-\zeta_{M_{Q}}^{(k)}\left(s_{0}+i\lfloor n \alpha\rfloor h, \mathbf{0}\right)\right|^{2}=\sum_{k=0}^{m-1} S_{k}^{\prime} .
$$


Let $k \in\{0, \ldots, m-1\}$. We apply Lemma 4 for

$$
\begin{gathered}
G(t)=\zeta^{(k)}\left(s_{0}+i t h\right)-\zeta_{M_{Q}}^{(k)}\left(s_{0}+i t h, \mathbf{0}\right): \\
S_{k}^{\prime} \leq \sum_{n=1}^{N}|G(\lfloor n \alpha\rfloor h)|^{2} \\
\leq \frac{1}{h(\alpha-1)} \int_{0}^{N \alpha h}|G(t)|^{2} \mathrm{~d} t+2\left(\int_{0}^{N \alpha h}|G(t)|^{2} \mathrm{~d} t\right)^{\frac{1}{2}}\left(\int_{0}^{N \alpha h}\left|G^{\prime}(t)\right|^{2} \mathrm{~d} t\right)^{\frac{1}{2}} .
\end{gathered}
$$

Using Lemma 1, we may choose $Q$ sufficiently large such that

$$
S_{1}<N \operatorname{meas}(K) \frac{\varepsilon^{3}}{2} \quad \text { for } N \geq N_{1}=N_{1}(Q) .
$$

Consequently, by (4), (7) and (8), we have

$$
A_{N}<\operatorname{meas}(K) \varepsilon^{3} \text { for } N>N_{2}=N_{2}(Q) .
$$

Since the sequence

$$
\left(\left\{\frac{h \ln p_{1}^{\prime}}{2 \pi}\lfloor n \alpha\rfloor\right\}, \ldots,\left\{\frac{h \ln p_{\ell}^{\prime}}{2 \pi}\lfloor n \alpha\rfloor\right\}\right)_{n \in \mathbb{N}}
$$

is u.d. $\bmod 1$ in $\mathbb{R}^{\ell}, A_{N}$ contains $\sim N$ meas $(K)$ terms in $\sum_{n=1}^{\prime N}$ as $N \rightarrow \infty$. Hence there exists an $n$ such that

$$
\left.\begin{array}{r}
\sum_{k=0}^{m-1}\left|\zeta^{(k)}\left(s_{0}+i\lfloor n \alpha\rfloor h\right)-\zeta_{M}^{(k)}\left(s_{0}+i\lfloor n \alpha\rfloor h, \mathbf{0}\right)\right|^{2}<\varepsilon^{3}, \\
\left(\left\{\frac{h \ln p_{1}^{\prime}}{2 \pi}\lfloor n \alpha\rfloor\right\}, \ldots,\left\{\frac{h \ln p_{\ell}^{\prime}}{2 \pi}\lfloor n \alpha\rfloor\right\}\right) \in K .
\end{array}\right\}
$$

Combining (3) and (9) we showed that there exists an $n$ such that

$$
\left|\zeta^{(k)}\left(s_{0}+i\lfloor n \alpha\rfloor h\right)-a_{k}\right|<3 \varepsilon
$$

for $k=0, \ldots, m-1$

The proof of the first part of Theorem 4 consists of the same arguments as we used until now. Instead of Lemma 3 we use Lemma 2, and there is no need to apply Leibniz's formula and the Cauchy-Schwarz inequality. 


\section{ATHANASIOS SOURMELIDIS}

Proof of The orem 5. Let $h \in L(\alpha) \cap[0,+\infty)$ and $\varepsilon>0$. Since the Taylor expansion of $g$ is valid for all $s \in K$, there exists an $N=N(\varepsilon)$ such that

$$
\max _{s \in K}\left|g(s)-\sum_{k=0}^{N-1} \frac{g^{(k)}\left(s_{0}\right)}{k !}\left(s-s_{0}\right)^{k}\right|<\frac{\varepsilon}{3} .
$$

From Theorem 4 , for the vector $\left(g\left(s_{0}\right), \ldots, g^{(N-1)}\left(s_{0}\right)\right)$ and $\varepsilon>0$, there exists a sequence $\left(n_{\ell}\right)_{\ell \in \mathbb{N}}$ such that for every $\ell=1,2, \ldots$ and every $k=0, \ldots, N-1$,

$$
\left|\zeta^{(k)}\left(s_{0}+i\left\lfloor n_{\ell} \alpha\right\rfloor h\right)-g^{(k)}\left(s_{0}\right)\right|<\varepsilon^{\prime}=\frac{\varepsilon}{3 N} \min _{0 \leq k \leq N-1} \frac{k !}{r^{k}} .
$$

We choose an $n_{\ell_{0}}=n_{\ell_{0}}(\varepsilon, h)$ such that $1 \notin K+i\left\lfloor n_{\ell_{0}} \alpha\right\rfloor h$. Then,

$$
\begin{array}{r}
\max _{s \in K}\left|\sum_{k=0}^{N-1} \frac{\zeta^{(k)}\left(s_{0}+i\left\lfloor n_{\ell_{0}} \alpha\right\rfloor h\right)}{k !}\left(s-s_{0}\right)^{k}-\sum_{k=0}^{N-1} \frac{g^{(k)}\left(s_{0}\right)}{k !}\left(s-s_{0}\right)^{k}\right| \\
\max _{s \in K} \varepsilon^{\prime} \sum_{k=0}^{N-1} \frac{\left|s-s_{0}\right|^{k}}{k !} \leq \frac{\varepsilon}{3} .
\end{array}
$$

The choice of $n_{\ell_{0}}$ allows us to represent $\zeta$ in the disk $K+i\left\lfloor n_{\ell_{0}} \alpha\right\rfloor$ as the sum of a Taylor series centered at $s_{0}+i\left\lfloor n_{\ell_{0}} \alpha\right\rfloor h$,

$$
\zeta\left(s+i\left\lfloor n_{\ell_{0}} \alpha\right\rfloor h\right)=\sum_{k=0}^{\infty} \frac{\zeta^{(k)}\left(s_{0}+i\left\lfloor n_{\ell_{0}} \alpha\right\rfloor h\right)}{k !}\left(s-s_{0}\right)^{k},
$$

for all $s \in K$. If

$$
M=M(\varepsilon, h)=\max _{s \in K}\left|\zeta\left(s+i\left\lfloor n_{\ell_{0}} \alpha\right\rfloor h\right)\right| \quad \text { and } \quad \delta \in(0,1),
$$

then, using Cauchy's estimates, we get

$$
\left|\frac{\zeta^{(k)}\left(s_{0}+i\left\lfloor n_{\ell_{0}} \alpha\right\rfloor h\right)}{k !}\left(s-s_{0}\right)^{k}\right| \leq \frac{M k !}{r^{k}} \frac{\left|s-s_{0}\right|^{k}}{k !} \leq M \delta^{k},
$$

for all $s \in \overline{D\left(s_{0}, \delta r\right)}$. Hence,

$$
\begin{aligned}
\mid \zeta\left(s+i\left\lfloor n_{\ell_{0}} \alpha\right\rfloor h\right) & -\sum_{k=0}^{N-1} \frac{\zeta^{(k)}\left(s_{0}+i\left\lfloor n_{\ell_{0}} \alpha\right\rfloor h\right)}{k !}\left(s-s_{0}\right)^{k} \mid= \\
& \left|\sum_{k=N}^{\infty} \frac{\zeta^{(k)}\left(s_{0}+i\left\lfloor n_{\ell_{0}} \alpha\right\rfloor\right)}{k !}\left(s-s_{0}\right)^{k}\right| \leq M \frac{\delta^{N}}{1-\delta},
\end{aligned}
$$




\section{WEAK UNIVERSALITY THEOREM}

for all $s \in \overline{D\left(s_{0}, \delta r\right)}$. Combining relations (10), (11) and (12), we find

$$
\left|\zeta\left(s+i\left\lfloor n_{\ell_{0}} \alpha\right\rfloor h\right)-g(s)\right|<M \frac{\delta^{N}}{1-\delta}+\frac{\varepsilon}{3}+\frac{\varepsilon}{3},
$$

for all $s \in \overline{D\left(s_{0}, \delta r\right)}$. Now choose $\delta=\delta(\varepsilon, h) \in(0,1)$ such that

$$
M \frac{\delta^{N}}{1-\delta}=\frac{\varepsilon}{3}
$$

This is possible since for the continuous function

$$
F:(0,1) \rightarrow \mathbb{R} \quad \text { with } \quad F(t)=M \frac{t^{N}}{1-t}, \quad t \in(0,1),
$$

we have

$$
\lim _{t \rightarrow 0} F(t)=0 \quad \text { and } \quad \lim _{t \rightarrow 1} F(t)=+\infty .
$$

We thus have shown

$$
\max _{\left|s-s_{0}\right| \leq \delta r}\left|\zeta\left(s+i\left\lfloor n_{\ell_{0}} \alpha\right\rfloor h\right)-g(s)\right|<\varepsilon
$$

and this completes the proof.

\section{REFERENCES}

[1] BAGCHI, B.: The Statistical Behaviour and Universality Properties of the Riemann Zeta-function and Other Allied Dirichlet Series, Thesis, Indian Statistical Institute, Calcutta, 1981.

[2] BOHR, H.: Zur Theorie der Riemannschen Zetafunktion im kritischen Streifen, Acta Math. 40 (1915), 67-100.

[3] BOHR, H.-COURANT, R.: Neue Anwendungen der Theorie der Diophantischen Approximationen auf die Riemannschen Zetafunktion, J. reine Angew. Math. 144 (1914), 249-274.

[4] CARLSON, D.: Good Sequences of Integers, Thesis, University of Colorado, 1971.

[5] GARUNKŠTis, R.-LAURINČIKAS, A.-MATSUMOTO, K.-STEUDING, J.-STEUDING, R.: Effective uniform approximation by the Riemann zeta-function, Pub. Mat., Barc. 54 (2010), 209-219.

[6] KUIPERS, L.-NIEDERREITER, H.: Uniform Distribution of Sequences. John Wiley \& Sons, New York, 1974. Reprint edition: Dover Publications, Inc. Mineola, New York, 2006. 


\section{ATHANASIOS SOURMELIDIS}

[7] REICH, A.: Werteverteilung von Zetafunktionen, Arch. Math. 34 (1980), 440-451.

[8] VORONIN, S. M.: On the distribution of nonzero values of the Riemann $\zeta$-function, Poc. Steklov Inst. Math 128 (1972), 153-175; translation from Trudy Mat. Inst. Steklov 128 (1972), 131-150.

Received April 15, 2016

Accepted February 22, 2017

\begin{abstract}
Athanasios Sourmelidis
Institute for Mathematics Chair of Mathematics IV

University of Würzburg

Campus Hubland Nord

Emil-Fischer-Straße 40

97074 Würzburg

GERMANY
\end{abstract}

E-mail: athanasios.sourmelidis

@mathematik.uni-wuerzburg.de 